\title{
Enfance
}

http://www.necplus.eu/ENF

Additional services for Enfance:

Email alerts: $\underline{\text { Click here }}$

Subscriptions: $\underline{\text { Click here }}$

Commercial reprints: $\underline{\text { Click here }}$

Terms of use : $\underline{\text { Click here }}$

\section{Les compléments neurophysiologiques du diagnostic}

C. Barthélémy, M. Huc-Chabrolles, G. Tripi, M. Gomot, J. Martineau et F. BonnetBrilhault

Enfance / Volume 2009 / Issue 01 / March 2009, pp 89 - 92

DOI: 10.4074/S0013754509001098, Published online: 16 April 2009

Link to this article: http://www.necplus.eu/abstract S0013754509001098

How to cite this article:

C. Barthélémy, M. Huc-Chabrolles, G. Tripi, M. Gomot, J. Martineau et F. Bonnet-

Brilhault (2009). Les compléments neurophysiologiques du diagnostic. Enfance, 2009, pp 89-92 doi:10.4074/S0013754509001098

Request Permissions : $\underline{\text { Click here }}$ 


\section{La boîte à outils du diagnostic d'autisme}





\title{
Les compléments neurophysiologiques du diagnostic
}

\author{
C. Barthélémy*, M. Huc-Chabrolles, G. Tripi, \\ M. Gomot, J. Martineau et F. Bonnet-Brilhault
}

\begin{abstract}
RÉSUMÉ
Les recherches présentées mettent en évidence des relations entre anomalies comportementales et cognitives et dysfonctionnements cérébraux sous-jacents à partir de méthodes d'exploration électrophysiologique non invasives (électroencéphalogramme, potentiels évoqués auditifs). Trois types de troubles sont étudiés : les troubles du sommeil, l'intolérance au changement et l'exploration visuelle atypique des visages humains. La complémentarité des approches cliniques et neurophysiologiques est cruciale aux étapes du diagnostic fonctionnel, de l'intervention thérapeutique et éducative.
\end{abstract}

MotS-CLÉs : AUTISME, EEG DE SOMMEIL, MÉLATONINE, POTENTIELS ÉVOQUÉS AUDITIFS, SYSTĖME DE SUIVI DU REGARD

\begin{abstract}
Neurophysiological complements of autism diagnosis

Research presented show relationships between behavioral and cognitive disorders and underlying cerebral functional abnormalities, on the basis of non invasive electrophysiological investigations (electroencephalography, cortical evoked potentials). Three types of disturbances are studied: sleep problems, intolerance to change and atypical visual processing of human faces. The complementarity of clinical and neurophysiological approaches is crucial at the levels of functional diagnosis, therapeutic and educative interventions.
\end{abstract}

KEY-WORDS: AUTISM, SLEEP EEG, MELATONIN, AUDITORY-EVOKED POTENTIALS, EYE-TRACKING SYSTEM

Les caractéristiques comportementales qui définissent l'autisme apparaissent liées à des dysfonctionnements neurophysiologiques centraux qui s'expriment par des déficits de décodage de l'information dite " sociale » et correspondent probablement à des anomalies de processus de régulations de base, en particulier de l'attention, de la perception, de la motricité, de l'émotion. Lelord (1990) a posé, très tôt, l'hypothèse «d'insuffisances modulatrices cérébrales » dans l'autisme.

Récemment, cliniciens et chercheurs mettent l'accent sur certains troubles classiquement considérés comme associés à l'autisme - les troubles du sommeil par exemple - ou inclus dans la triade symptomatique de ce syndrome : l'intolérance au changement et l'exploration visuelle atypique des visages. 
Les résultats présentés sont pour certains préliminaires. Ils mettent en évidence des relations entre anomalies du comportement dans la vie quotidienne des personnes avec autisme et des particularités neurofonctionnelles ouvrant sur des modèles neurobiologiques, génétiques et $d$ 'intéressantes perspectives thérapeutiques et éducatives.

\section{TROUBLES DU SOMMEIL - ASPECTS NEUROPHYSIOLOGIQUES ET THÉRAPEUTIQUES}

Les perturbations du sommeil, jusqu'à maintenant non explorées systématiquement chez les personnes avec autisme dans la clinique de routine se révèlent très fréquentes, durables et handicapantes. Elles touchent gravement la qualité et la quantité du sommeil. Dans certains cas comme celui présenté, il y a inversion dans la répartition circadienne du sommeil et de l'éveil : peu ou pas de sommeil la nuit, endormissement dans la journée.

Le protocole thérapeutique développé par notre équipe (Huc-Chabrolle et al., 2009) comprend :

- l'observation clinique approfondie,

- l'enregistrement polysomnographique du sommeil,

- le dosage plasmatique de la mélatonine (cycle circadien), avant, pendant et après traitement par la mélatonine (circadin).

Les résultats recueillis pour A.A., jeune homme de dix-sept ans, avec autisme atypique, montre que la désorganisation du sommeil enregistré sur l'hypnogramme correspond à une absence de pic nocturne plasmatique de mélatonine. Ces anomalies, largement corrigées sous traitement, réapparaissent à l'arrêt de celui-ci ainsi que l'insomnie et les troubles de vigilance et du comportement diurne. Cette observation est à confirmer sur un groupe suffisamment large de patients.

De plus, la confrontation des données de cette étude avec les observations et hypothèses de l'équipe de T. Bourgeron (Melke et al., 2008) peut ouvrir sur des perspectives physiopathologiques et pharmacologiques prometteuses.

\section{INTOLÉRANCE AU CHANGEMENT - DU PHÉNOTYPE CLINIQUE ET ÉLECTROPHYSIOLOGIQUE AU GÉNOTYPE}

Le caractère restreint et répétitif de l'activité cognitive et comportementale fait partie de la triade des critères de diagnostic d'autisme. À l'heure actuelle, l'intérêt des chercheurs s'accroît pour l'exploration de ces particularités de fonctionnement (Bourreau et al., 2008) qui peuvent être très gênantes et résistantes aux interventions éducatives et thérapeutiques actuellement disponibles.

Les résultats publiés par Gomot et al. (2006) montrent qu'il existe un lien entre intolérance au changement et réactivité anormale du cortex 
cingulaire antérieur gauche aux événements inhabituels. Ces relations cliniques et neurofonctionnelles sont à la base de travaux en cours sur l'identification de "marqueurs " électrophysiologiques des dysfonctionnements autistiques.

Ces variables " phénotypiques " sont d'un intérêt majeur pour la constitution de sous-groupes homogènes indispensables pour les avancées futures de la recherche en génétique. Les résultats préliminaires en ce jour ont été obtenus au sein d'une famille dont plusieurs membres déficients mentaux avec ou sans autisme sont porteurs de la même mutation génétique NLG4 (Laumonnier et al., 2004).

\section{EXPLORATION VISUELLE ATYPIQUE DES VISAGES - ÉTUDE DÉVELOPPEMENTALE}

Dans le champ maintenant immense des recherches sur le fonctionnement normal et pathologique du cerveau dit "social », il est très important de disposer de données comportementales fiables caractérisant les personnes avec autisme tout au long de la vie. Les nouvelles technologies, celles de l'eye-tracking en particulier, nous permettent d'explorer dans un contexte naturel et motivant les stratégies d'exploration visuelle des scènes sociales et des visages en particulier. Les résultats obtenus par Hernandez et al. (2008) montrent que les stratégies de balayage visuel de la photographie d'un visage sont différentes chez les enfants et les adultes avec autisme comparés à des témoins de même âge en bonne santé. De plus, les temps de fixation $\mathrm{du}$ regard sur les zones du visage très riches en information sociale, les yeux par exemple, sont beaucoup moins longs chez les enfants avec autisme tout au cours du développement. Ces différences persistent chez l'adulte.

Ces variables psychophysiologiques sont très précieuses pour caractériser des comportements sociaux à mettre en relation avec des fonctionnements cérébraux (Martineau et al., 2008). Elles sont d'ores et déjà utilisables dans les études de suivi pour évaluer les effets des thérapies de la communication par exemple. Enfin, elles éclairent sur des fonctionnements-cibles à exercer pour faciliter les interactions sociales et la communication des personnes avec autisme dès le plus jeune âge (Barthélémy et al., 1995 ; Blanc et al., 2005).

\section{Conclusion}

Les progrès technologiques en exploration du comportement (vidéo, eye-tracking) ainsi qu'en imagerie cérébrale fonctionnelle (électrophysiologique et par résonance magnétique) permettent d'appliquer dans la pratique d'évaluation diagnostique au quotidien et dans la recherche des explorations non invasives pour l'étude dynamique du fonctionnement et du développement cérébraux. Ces 
hypothèses «neuronales » de l'autisme rejoignent celles des psychologues qui ont modélisé les particularités de fonctionnement cognitif, social, dans cette pathologie. La complémentarité des approches cliniques et neurophysiologiques est cruciale à l'étape du diagnostic fonctionnel mais aussi et surtout à celle de la prise en charge éducative et thérapeutique.

\section{Bibliographie}

Barthélémy, C., Hameury, L., Lelord, G. (1995). L'autisme de l'enfant, la Thérapie d'Echange et de Développement. Elsevier (ed.), Paris, 396 p.

Blanc, R., Adrien, J.L., Thiebaut, E.,et al. (2005). Bases neuropsychologiques des interactions sociales et des émotions dans l'autisme : de l'évaluation à la thérapeutique. In : Hommet, C., Jambaqué, I., Billard, C., Gillet, P. (ed.), Neuropsychologie de l'enfant et troubles du développement (pp. 389-402), Marseille : Solal Editeurs.

Bourreau, Y., Roux, S., Gomot, M., Barthélémy, C. (2009). Comportements répétés et restreints $(\mathrm{C} 2 \mathrm{R})$ dans les troubles autistiques : évaluation clinique. L'encéphale, sous presse.

Gomot, M., Bernard, F.P., Davis, M.H. et al. (2006). Change detection in children with autism : an auditory event-related fMRI study. Neuroimage, 29 (2), 475-484.

Hernandez, N., Metzger, A., Magne, R. et al. (2008). Exploration of core features of a human face by healthy and autistic adults analysed by visual scanning. Neuropsychologia, doi:10.1016:j.neuropsychologia, 1002.

Huc-Chabrolle, M., Malpaux, B Lucas, B., et al. (2009). Biological and polysomnographic exploration of sleep in ASD: clinical observations and therapeutic assessment. En preparation.

Laumonnier, F, Bonnet-Brilhault, F, Gomot, M, et al. (2004). X-linked mental retardation and autism are associated with a mutation in the NLGN4 gene, a member of the neuroligin family. Am J Hum Genet. Mar ; 74 (3):552-7.

Lelord, G. (1990). Physiopathologie de l'autisme - Les insuffisances modulatrices cérébrales, Neuropsychiatrie de l'Enfance, 3 (1-2), 43-49.

Martineau, J., Cochin, S., Magné, R., et al. (2008). Impaired cortical activation in autistic children: Is the mirror neuron system involved? Int. J. Psychophysiol., 68 (1), 35-40.

Melke, J, Goubran Botros, H, Chaste, P, et al. (2008). Abnormal melatonin synthesis in autism spectrum disorders. Mol Psychiatry. Jan ; 13 (1): 90-8. 